\section{On the assessment of arterial compliance from carotid pressure}

Title page waveform

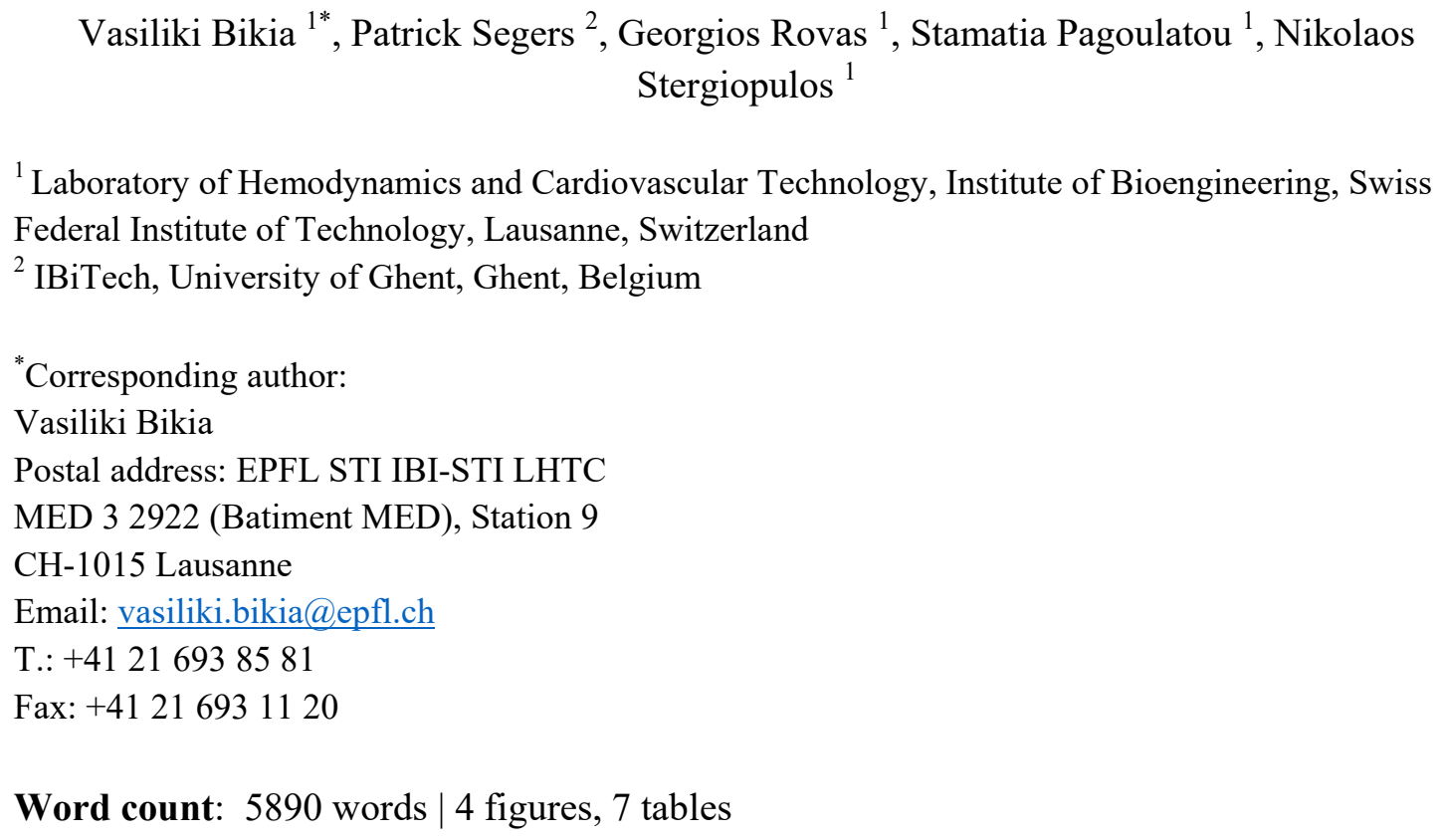

\section{Abstract}

In a progressively aging population, it is of utmost importance to develop reliable, noninvasive, and cost-effective tools to estimate biomarkers which can be indicative of cardiovascular risk. Various pathophysiological conditions are associated to changes in the total arterial compliance $\left(\mathrm{C}_{\mathrm{T}}\right)$, and thus its estimation via an accurate and simple method is valuable. Direct noninvasive measurement of $\mathrm{C}_{\mathrm{T}}$ is not feasible in the clinical practice. Previous methods exist for indirect estimation of $\mathrm{C}_{\mathrm{T}}$, which, however, require noninvasive, yet complex and expensive, recordings of the central pressure and flow. Here, we introduce a novel, noninvasive method for estimating $\mathrm{C}_{\mathrm{T}}$ from a single carotid waveform measurement using regression analysis. Features were extracted from the carotid wave and are combined with demographic data. A prediction pipeline is adopted for estimating $\mathrm{C}_{\mathrm{T}} \mathrm{using}$, firstly, a feature-based regression analysis and, secondly, the raw carotid pulse wave. The proposed methodology was appraised using the large human cohort $(n=2256)$ of the Asklepios study. Accurate estimates of $\mathrm{C}_{\mathrm{T}}$ were yielded for both prediction schemes; namely, $\mathrm{r}=0.83$ and normalized-RMSE $=9.58 \%$ for the feature-based model, and $\mathrm{r}=0.83$ and normalizedRMSE $=9.67 \%$ for the model which used the raw signal. The major advantage of this method pertains to the simplification of the technique offering easily applicable and convenient $\mathrm{C}_{\mathrm{T}}$ monitoring. Such an approach could offer promising applications, ranging from fast and costefficient hemodynamical monitoring by the physician to integration in wearable technologies. 
44 Keywords: vascular ageing, noninvasive monitoring, machine learning, artificial neural

45 network, pulse wave analysis

\section{New \& Noteworthy}

This paper introduces a novel artificial intelligence method to estimate total arterial compliance $\left(\mathrm{C}_{\mathrm{T}}\right)$ via exploiting the information provided by an uncalibrated carotid blood pressure waveform as well as typical clinical variables. The major finding of this study is that, $\mathrm{C}_{\mathrm{T}}$, which is usually acquired using both pressure and flow waveforms, can be accurately derived by the use of the pressure wave alone. This method could potentially facilitate easily applicable and convenient monitoring of $\mathrm{C}_{\mathrm{T}}$.

\section{Introduction}

In a progressively aging population, it is of utmost importance to develop reliable, noninvasive, and cost-effective tools for estimating relevant biomarkers which can be indicative of cardiovascular risk. Numerous invasive and non-invasive markers have been researched, but there is still the need for additional structural and functional parameters that would be able to assess cardiovascular risk [1]. The total arterial compliance $\left(\mathrm{C}_{\mathrm{T}}\right)$ is a biomechanical property of the arterial tree with great physiological and pathological importance [2]-[4]. $\mathrm{C}_{\mathrm{T}}$ and peripheral resistance constitute a major part of the arterial load on the heart [5]. Arterial compliance expresses the ability of the arterial system to store blood during systole without excessive pressure rise, and influences central blood pressure [6] and stroke volume [7]. The $\mathrm{C}_{\mathrm{T}}$ is becoming a promising parameter for evaluating assessment of the relationship between structural and functional changes in the vascular system with respect to its elasticity and capacity [8], [9]. Alterations in arterial compliance are associated to various physiological (aging) [10] or pathological (hypertension) conditions [11], which cannot be necessarily assessed by current biomarkers. Importantly, $\mathrm{C}_{\mathrm{T}}$ has been found to be superior over traditional evaluation techniques including pulse pressure and echocardiography [9], [11]. In addition, other studies have shown that $\mathrm{C}_{\mathrm{T}}$ was proven capable of differentiating among diseased, elderly, and health individuals [10]-[12]. In view of the emerging evidence on the importance of $\mathrm{C}_{\mathrm{T}}$ [8], the development of an accurate and simple method for its estimation may be valuable.

Direct noninvasive measurement of $\mathrm{C}_{\mathrm{T}}$ is not feasible in the clinical practice. Several methods have been proposed for indirect estimation of $\mathrm{C}_{\mathrm{T}}$ [13]-[16]. Most commonly, these methods require simultaneous recordings of the proximal aortic pressure and flow waves. Some of the most reliable and accurate techniques include the decay time method (DTM), the area method (AM), and the pulse pressure method (PPM), [15], [17]. The principle of the DTM is that during diastole there is no inflow from the heart, and thus, the decrease of aortic pressure, is characterized by the decay time. This decay can be fitted monoexponentially to any portion of the diastole to yield the characteristic time or time constant, which is $\mathrm{RC}_{\mathrm{T}}$, where $\mathrm{R}$ is a known value of peripheral resistance [17]. The AM was introduced by Randall 
88 [18] and it essentially represents an integral variation of the exponential decay method.

89 Compliance is calculated from $\mathrm{RC}_{\mathrm{T}}=\int_{t 1}^{t 2} P d x /\left(P_{1}-P_{2}\right)$, where $\mathrm{P}_{1}$ and $\mathrm{P}_{2}$ are diastolic

90 pressure at time points $t_{1}$ and $t_{2}$, respectively. The PPM is based on the fact that the modulus

91 of the input impedance of the arterial system is represented very well by the two-element

92 Windkessel model for the low frequencies $1^{\text {st }}$ to $5^{\text {th }}$ harmonic). Therefore, the pulse pressure

93 will be similar in the true arterial system and the two-element Windkessel model. The PPM

94 uses an iterative process that yields the value of $\mathrm{C}_{\mathrm{T}}$ that gives the best fit between the

95 measured pulse pressure and the pulse pressure predicted by the two-element Windkessel 96 model.

\section{2. Materials and Methods}

Yet, the invasive nature, lack of convenience or high cost of the required measurements have limited the assessment of $\mathrm{C}_{\mathrm{T}}$, namely the inverse of arterial stiffness, in every day clinical practice, while surrogates of local or regional arterial stiffness have been applied commonly [16], [19]. Measurement of carotid-femoral pulse wave velocity (cfPWV) is considered as the gold standard to evaluate arterial stiffness [20].

Recent advances in Machine Learning have expanded the areas and the opportunities in developing novel modelling and predictive methods for clinical use [21]. In a previous study, Tavallali et al. proposed and validated a method for estimating cfPWV from the carotid waveform and clinical parameters using neural networks [22]. Their results showed that this approach can provide accurate estimates of cfPWV. This offers an advancement in the assessment of arterial stiffness via cfPWV.

In view of these nascent opportunities, the present study introduces a novel, noninvasive, cost-efficient method for estimating $\mathrm{C}_{\mathrm{T}}$ from a single carotid waveform measurement using regression analysis. The proposed methodology uses an uncalibrated carotid blood pressure waveform which is subsequently calibrated using the brachial blood pressure values. Features are extracted from the carotid wave and are combined with readily available clinical parameters such as age, gender, height, weight. A prediction pipeline is adopted for estimating $\mathrm{C}_{\mathrm{T}}$ using, firstly, a feature-based regression analysis and, secondly, the raw carotid pulse wave. A main advantage of this method pertains to the avoidance of aortic blood flow recording which is commonly required by prior $\mathrm{C}_{\mathrm{T}}$ estimators. Given that accurate values of $\mathrm{C}_{\mathrm{T}}$ are cumbersome to obtain in the intact organism, in this study, the accuracy of the predictive model was evaluated by comparing the predictions against the $\mathrm{C}_{\mathrm{T}}$ values which were derived using the precise and extensively validated PPM [14], [15], [23].

\subsection{Asklepios database}

Human data were available from the Asklepios study, a broad prospective longitudinal study with the aim of assessing the development and progression of cardiovascular disease [24]. A total of 2404 subjects were found eligible to be included in the study. The participants underwent a noninvasive evaluation of central hemodynamics, including recordings of carotid blood pressure and aortic blood flow waveforms. The inclusion and exclusion criteria are listed in Table 1. The study protocol was approved by the ethical committee of Ghent University Hospital and informed consent of participation was given by all subjects. A 
comprehensive description of the Asklepios data can be found in the original publication [24].

\subsection{Measurement of pressure and flow waves}

Blood pressure recordings were performed at the left common carotid artery via applanation tonometry using a Millar pen-type tonometer (SPT 301; Millar Instruments, Houston, Texas, USA). The measurement set-up, processing, and calibration procedure (based on sphygmomanometer systolic and diastolic blood pressure and applanation tonometry at the brachial artery) were previously described in detail [24], [25].

The carotid pressure was derived as a "mean" waveform of multiple beats from a 20second recording [6]. Pressure data were recorded in continuous sequences of 20 seconds. The postprocessing included signal filtering (Savitsky-Golay filter, Matlab, The Mathworks Inc.). Subsequently, identification of individual cycles, detrending (i.e., linearly smoothing out eventual differences in the numerical value of the start and end of the cycle), and averaging were performed. The cycles with a cycle length shorter or longer than $20 \%$ of the mean cycle duration were automatically deselected. The same applied for cycles with a shape surpassing the "envelope" curves, which were constructed from the average \pm (two times the standard deviation). The process was repeated iteratively until all cycles were within the "envelope" curves. As an arbitrary quality criterion, data were accepted only if minimally 10 cycles were retained. The average of these cycles was considered as the tonometry recording for the carotid artery. The carotid waveform was calibrated by assuming that diastolic and mean BP values remain fairly constant for the major arteries.

Flow at the aorta was measured using ultrasound (VIVID 7; GE Vingmed Ultrasound, Horten, Norway) from the cross-sectional area and blood velocities in the left ventricular outflow tract (LVOT). The internal diameter of the LVOT was measured in the parasternal long-axis view at the valve annulus, and LVOT area was calculated assuming a circular cross-section. Flow velocities were obtained in the LVOT via pulsed wave Doppler in the apical 5-chamber view. Images were exported in raw DICOM format and processed off-line within a dedicated software interface in Matlab (The Mathworks, Natick, MA). For each cardiac cycle, the onset and end of systolic ejection were visually delineated with two cursors, after which the contours in the systolic phase were automatically traced using the transition in pixel intensity above a user-defined threshold value. Two to three cycles were averaged, and the average cycle sub-sampled to 500 sample points and smoothed using a Savitsky-Golay filter (order 3, frame width 31). The maximal velocities were multiplied with the LVOT cross-sectional area to obtain the aorta flow waveform (assuming a flat velocity profile in the LVOT). This approach yielded physiologically relevant values of stroke volume and cardiac output [2].

The heart rate (HR) was calculated from the average duration of pressure and flow signals. The time vectors of the two signals were normalized, synchronized and then denormalized, rendering the heart cycle length equal to the average length of the pressure and flow waveform.

\subsection{Derivation of the reference compliance}

Measurement of the real $\mathrm{C}_{\mathrm{T}}$ values in a human cohort is not feasible. In the present study, the pulse pressure method (PPM) was used as the ground truth value for compliance [23]. The PPM is based on the fact that the modulus of the input impedance of the arterial system is represented very well by the two-element WK model for the low frequencies $\left(1^{\text {st }}\right.$ to $5^{\text {th }}$ harmonic). Therefore, the pulse pressure will be similar in the true arterial system and the 
two-element WK model. From the ratio of mean pressure over mean flow we derive peripheral resistance. Then, using measured flow as input to the two-element WK, the predicted pulse pressure is fit to the actual pulse pressure by adjusting compliance. Compliance adjustment is done by a simple "trial and error" type of approach knowing that lower compliance yields larger pulse pressures. Following an iterative process, the value of the compliance $\left(\mathrm{C}_{\mathrm{T}}\right)$ that gives the best fit of the measured pulse pressure provides the estimate of the compliance. The method has been thoroughly validated both in-silico under various hemodynamical states [17], [23] and against in-vivo data [14] and it has been proven to be capable of accurately estimating arterial compliance.

\subsection{Features extraction from the carotid pressure wave}

The features were extracted from the carotid pressure signal and its time derivative (Figure 1). Concretely, the features include the systolic blood pressure (SBP), the diastolic blood pressure $(\mathrm{DBP})$, the dicrotic notch pressure point $\left(\mathrm{P}_{\mathrm{DN}}\right)$, the dicrotic notch time point $\left(\mathrm{t}_{\mathrm{DN}}\right)$, the upstroke systolic area $\left(\mathrm{A}_{\text {upstroke }}\right)$, the total systolic area $\left(\mathrm{A}_{\text {systolic }}\right)$, the diastolic area $\left(\mathrm{A}_{\text {diastolic }}\right)$, the peak of time derivative $\left(\mathrm{dP} / \mathrm{dt}_{\max }\right)$, the time point that peak derivative occurs $\left(\mathrm{t}_{\mathrm{dP} / \mathrm{dtmax}}\right)$, and the heart rate $(\mathrm{HR})$.

\subsection{Regression Analysis}

The extracted features, i.e., SBP, DBP, MAP, PP, $\mathrm{P}_{\mathrm{DN}}, \mathrm{t}_{\mathrm{DN}}, \mathrm{A}_{\text {upstroke }}, \mathrm{A}_{\text {systolic }}, \mathrm{A}_{\text {diastolic}}$, $\mathrm{dP} / \mathrm{dt}_{\max }, \mathrm{t}_{\mathrm{dP} / \mathrm{dtmax}}, \mathrm{HR}$, as well as demographic data including age, gender, height, weight were used as the input features to the ML model. The $\mathrm{C}_{\mathrm{T}}$ (as derived from PPM) was set to be the target output variable. The data were organized in pairs (inputs-outputs) and were kept for the training/testing process. For the regression process, we used an artificial neural network (ANN) and a Linear Regressor to estimate the target variable of interest. Furthermore, the performance of a predictive model including cardiac output (CO) as an additional input feature was assessed. It should be noted that the models including the $\mathrm{CO}$ feature are not considered as the main focus of the present study. We, however, decided to include them in the analysis for investigating the importance of $\mathrm{CO}$ in the estimations. For the ANN, a fixed one-hidden layer structure was selected and the "Adam" optimizer was used [26]. In addition, the ANN was trained/tested using as input the entire raw carotid waveform, as well as demographic data. The carotid BP waveforms were sampled at 100 data points per cycle. The predictive models are summarized in Table 2.

One-hundred forty eight out of the 2404 participants were excluded due to missing or erroneously measured data. Their exclusion led to a final size population equal to 2256 participants. The data were randomly split into three sets: a training set $(80 \%$ of the total data set), a validation set (10\%) and a test set (10\%). Therefore, out of the entire cohort, 1796 subjects were used for the training, and 223 data instances were used as validation for the hyperparameter selection and 237 subjects were kept for the testing. To mitigate overfitting and to increase the generalization capacity, the model should be trained for optimal hyperparameter values. For the ANN, the batch_size (defines the number of samples that will be propagated through the network) was set to be equal to 200, while the number of epochs was optimized. The number of epochs defines the number of times that the learning algorithm works through the entire training dataset. For selecting the optimal value for epochs, we 
computed the train loss and the validation loss for various values of epochs. Here, the loss corresponds to the mean square error (MSE). Loss values were monitored by Early stopping call back function. When an increment is observed in the loss values, training comes to halt and the respective value of epoch indicates the optimal selection. All the yielded optimized hyperparameters are aggregated in Table 3. Subsequently, the test set was fed into the trained models to predict $\mathrm{C}_{\mathrm{T}}$ and the precision was evaluated.

The current study aimed to evaluate the importance of each input feature for the $\mathrm{C}_{\mathrm{T}}$ prediction. The importance was quantified by the use of the permutation feature importances [27]. The concept of permutation feature importances relies on measuring the importance of a feature by calculating the increase in the prediction error after permuting the feature. The permutation importances were computed by shuffling the values of each feature on the test set and by estimating the RMSE after the permutation. This process was repeated 20 times and the mean and standard deviation of the increase in RMSE were reported. Subsequently, an additional ANN was trained/tested using the 5 most important features yielded by the aforementioned analysis. The training/testing pipeline as well as the pre- and post-analyses were implemented using the Scikit-learn library [28] in a Python programming environment. The pandas and numpy packages were also used [29], [30].

\subsection{Sensitivity to noise and variations in the wave morphology}

We further evaluated the robustness of the method in the case of measurement noise or variations in the morphology of the wave. The evaluation was done following two controlled experiments. Firstly, errors were considered for the extraction of the wave-based features for ANN1 model. Concretely, errors in features were simulated with a random distribution. The error for each variable was randomly drawn from the range of $[-5,5] \%$ (simulating a maximum noise level equal to $+5 \%$ ). Subsequently, each variable value was multiplied with a noise factor; for instance, for a randomly selected error of $-4 \%$, the respective variable value was multiplied with a noise factor equal to 0.96 . The process was repeated for the noise ranges of \pm 7 and $\pm 10 \%$. Secondly, we wished to simulate adverse effects for the ANN3 by distorting the shape of the input pressure wave. This was achieved by adding white gaussian noise assuming three signal-to-ratio (SNR) values, i.e., 40, 35, and $30 \mathrm{~dB}$. Selection of the SNR values was done experimentally so that an obvious distortion in the wave morphology is achieved that could render the model incapable of making a correct prediction. A lower SNR value would lead to an unrealistically signal variation which would be inappropriate to use and thus would be discarded. A higher SNR value would make it easy for the model to yield a precise estimation.

\subsection{Statistical analysis}

All data are presented as mean and standard deviation (SD). The statistical analysis was performed in Python (Python Software Foundation, Python Language Reference, version 3.6.8, available at http://www.python.org). The accuracy between the estimates and the reference values were evaluated by using the Pearson's correlation coefficient ( $r$ ), the normalized root mean square error (nRMSE). Bias and limits of agreement (LoA) (where the $95 \%$ of errors are expected to lie) were calculated using the Bland-Altman analysis [31]. The computed nRMSE was based on the difference between the minimum and maximum values 
of the dependent variable $\mathrm{y}$ and was computed as $\mathrm{RMSE} /\left(\mathrm{y}_{\max }-\mathrm{y}_{\min }\right)$. Level of statistical significance was set equal to 0.05 .

\section{Results}

The population consisted of 1087 (48\%) male participants and 1169 (52\%) female participants. The distributions of the cardiovascular parameters of the study cohort $(n=2256)$ are presented in Table 4.

\subsection{Comparison between the model-predicted and reference data.}

The scatterplots and the Bland-Altman plots of the estimated $\mathrm{C}_{\mathrm{T}}$ for each of the models against the ground truth are shown in Figures 2 and 3. Regression metrics for the agreement, precision and bias are aggregated in Table 5. The regression slopes were similar for

the LR1, ANN1, and ANN3 in which the demographic data were considered as inputs. Accuracy was significantly increased for the models which used $\mathrm{CO}$ as an input feature $(\mathrm{r} \geq 0.94)$. Variability of the absolute errors between predicted and actual compliance values was low for the LR1, ANN1, and ANN3. In all models, LoA were narrow and biases were reported to be close to zero. Table 6 presents the feature importances of the input regressors for $\mathrm{C}_{\mathrm{T}}$, respectively. Among the inputs, PP, SBP, and $\mathrm{A}_{\text {diastolic }}$ appeared to have the highest importance levels (error increase was more than $0.20 \mathrm{~mL} / \mathrm{mmHg}$ ). On the other hand, $\mathrm{P}_{\mathrm{DN}}$ and $A_{\text {upstroke }}$ had the lowest importance levels (error increased by $0.01 \mathrm{~mL} / \mathrm{mmHg}$ ). The ANN5 using only the 5 top-contributing features had a satisfactory performance similar to the one of ANN1 which used all the extracted wave-based features and the performance of ANN3 which was fed with the entire waveform and the demographical data (nRMSE was found to be close to $10 \%$ and correlation equal to 0.82 ).

\subsection{Sensitivity to noise and variations in the wave morphology}

An input carotid pressure wave with the simulated artificial noise is illustrated in Figure 4. The addition of artificial noise affected the wave's shape, harming the smoothness of the curve and leading to variations in the peaks. We can observe that for an $\mathrm{SNR}=30 \mathrm{~dB}$, it begins to become difficult to clearly distinguish the shape. As anticipated, the agreement and correlation between the estimated $C_{T}$ and the reference $C_{T}$ decreased with the increase in the noise level. Table 7 reports the correlation coefficients and normalized RMSE values as a function of the noise levels for the two simulated experimental settings.

\section{Discussion}

This paper introduces a novel machine learning method for estimating $\mathrm{C}_{\mathrm{T}}$. The findings indicated that arterial compliance can be accurately predicted by exploiting the carotid blood pressure waveform. This method relies on the raw information hidden in the carotid pulse wave that can be unveiled via the sophisticated machine learning capacity. In addition, the present study introduces an ANN estimator which is based on features extracted from the carotid wave and appear to be powerful predictors of $\mathrm{C}_{\mathrm{T}}$. The major advantage of a 
309

310

311

312

313

314

315

316

317

318

319

320

321

322

323

324

325

326

327

328

329

330

331

332

333

334

335

336

337

338

339

340

341

342

343

344

345

346

347

348

349

350

351

352

method for estimating $\mathrm{C}_{\mathrm{T}}$ from a single carotid pressure waveform is that it eliminates the need for a flow or velocity recording which requires complex and expensive Echocardiographic or Magnetic Resonance Imaging procedures. Consequently, it provides a faster and more convenient way for monitoring arterial compliance.

The $\mathrm{C}_{\mathrm{T}}$ together with the total vascular resistance are the two major parameters that describe the global biomechanical properties of the arterial system. Modeling vasculature and hemodynamical responses often require the estimation of $\mathrm{C}_{\mathrm{T}}$, while other methods for minimally invasive cardiac output monitoring (namely, pulse contour analysis) are also dependent on $\mathrm{C}_{\mathrm{T}}$ values [32]. Yet, despite the additional clinical utility of $\mathrm{C}_{\mathrm{T}}$, current techniques for $\mathrm{C}_{\mathrm{T}}$ have not entered the everyday clinical practice. This is mainly attributed to inherent limitations, including methodological complexity and expensiveness.

Moreover, the lack of a common basis and guidelines has hampered the establishment of $\mathrm{C}_{\mathrm{T}}$ as an outcome predictor. However, several studies have demonstrated that assessment of $\mathrm{C}_{\mathrm{T}}$ is valuable not only for cardiovascular risk evaluation but also for assessment of the relationship between structural and functional changes in the vascular system with respect to its elasticity [14], [33]. Moreover, Haluska et al. stressed that derivation of $C_{T}$ adds incremental benefit to Framingham risk scores in patients with intermediate cardiovascular risk [9]. Hence, $\mathrm{C}_{\mathrm{T}}$ is becoming a valuable parameter in the clinical setting by providing additive value in conjunction with other vascular characteristics [12] or by acting as a superior predictor over current traditional techniques [9]. The suggested method could potentially facilitate the further elucidation of the clinical utility of $\mathrm{C}_{\mathrm{T}}$ as a risk predictor.

The current study trained and tested two ML models of different nature, namely, a typical linear regressor, and an artificial neural network. There was not a significant variability in the errors among the two models for the feature-based configurations, i.e., LR1 and

ANN1, (LoA were $[-0.35,0.35]$ and $[-0.33,0.34] \mathrm{mL} / \mathrm{mmHg}$ ). However, the LR1 could not account for the nonlinear relationships between the inputs and the compliance and this led to curvilinearity in the results' plots. Importantly, there is much additive value offered by the ANN estimator which has been proven capable of accurately predicting $\mathrm{C}_{\mathrm{T}}$ from the raw blood pressure waveform. This approach could introduce a greatly promising method for the medical community by reducing the cost and the complexity in assessing $\mathrm{C}_{\mathrm{T}}$. Moreover, as anticipated, the inclusion of $\mathrm{CO}$ in the input vector essentially increased the precision of the results. Compliance is a measure of volume change against unit pressure change. Hence, the two parameters are highly interdependent. The dependency in conjunction to the blood pressure information allows for computing the one from the other. This is a principal applied by several existing methods. In addition, providing that the PPM (used to derive the reference $\mathrm{C}_{\mathrm{T}}$ ) utilizes the aortic flow for the $\mathrm{C}_{\mathrm{T}}$ calculation, introducing the aortic flow information to the machine learning model would inarguably reduce the error. Nevertheless, our study's objective is to provide estimates of $\mathrm{C}_{\mathrm{T}}$ without the need for the aortic flow or velocity recording (and thus $\mathrm{CO}$ ).

It is of importance to recognize the contribution of each input to the prediction of $\mathrm{C}_{\mathrm{T}}$. The PP was found to have the highest influence on the prediction error, namely $0.31 \pm 0.04$ $\mathrm{mL} / \mathrm{mmHg}$ for $\mathrm{C}_{\mathrm{T}}$ values within a range of $0.3-2.1 \mathrm{~mL} / \mathrm{mmHg}$. This is highly anticipated given that PP is essentially determined by the elastic properties of the aorta [34]. Due to the 
353 topological proximity of the carotid artery to the aorta, the carotid PP constitutes a fair 354 surrogate of the aortic PP. Hence, the strong interdependence between the PP and $\mathrm{C}_{\mathrm{T}}$ is also 355 in effect for the carotid pressure. Moreover, one should not ignore the fact that the PPM, 356 which was applied for acquiring the compliance values, relies on an iterative process that 357 yields the $\mathrm{C}_{\mathrm{T}}$ with the best fit in terms of PP. The SBP also appeared to impact the accuracy 358 of the estimation by an error increase of $0.28 \pm 0.02 \mathrm{~mL} / \mathrm{mmHg}$. The PP and SBP were 359 followed by $\mathrm{A}_{\text {diastolic }}$ and $\mathrm{A}_{\text {systolic }}$. The combination of the latter yields the entire area under the 360 curve whose measurement is involved in the arterial pulse contour analysis for $\mathrm{CO}$ estimation 361 [32]; knowledge of MAP and a notion of CO allows for approximating arterial compliance. 362 Moreover, the substantial contribution of the $\mathrm{A}_{\text {diastolic }}$ may be attributed to its association to 363 the decay time constant $\left(\tau=\mathrm{RC}_{\mathrm{T}}\right)$ whose concept it is employed by the AM for estimating 364 compliance. The demographic information, and in particular weight, had a high importance 365 level for the $\mathrm{C}_{\mathrm{T}}$ estimator. This was also observed from the reduced precision of the 366 predictive model which excluded the individual's demographic data from the input vector 367 (ANN4) where correlation was decreased to 0.77. Arterial compliance has been shown to be 368 highly dependent on arterial geometry which is determined by the body size, and thus weight 369 and height. When only the most importance features were used in ANN5 (permutation 370 importance higher than $0.1 \mathrm{~mL} / \mathrm{mmHg}$ ), the accuracy was remained similarly high as the one 371 of the ANNs which used either all the extracted features (ANN1) or all the wave points 372 (ANN3). Therefore, it should not be necessary to use a higher number of input features for 373 the $\mathrm{C}_{\mathrm{T}}$ predictive models. Finally, the lower importance levels of some inputs might be 374 explained by the fact that the information embedded in their values may be contained already 375 in other inputs with higher importance levels.

376 Estimation of cfPWV using the proposed methodology would yield a correlation 377 equal to 0.6 between the estimated and the reference values (data not shown). It is likely that 378 the lower correlation is attributed to the fact that the method uses as input a waveform from a 379 single arterial site, while measurement of the foot-to-foot cfPWV requires waves from two 380 arterial locations. Nevertheless, cfPWV can be measured in an easy and noninvasive way 381 with satisfactory reproducibility, and hence further simplification of its acquisition would not 382 add tremendously to the current state of the art. In contrast, fast, convenient, and cost383 efficient determination of $\mathrm{C}_{\mathrm{T}}$ is still missing.

384 In this study, we chose to use a single carotid pressure waveform for estimating 385 arterial compliance. The rationale behind the use of a single wave relies on the current 386 function of the existing devices. The current commercial devices (e.g., SphygmoCor) collect 387 multiple recordings of the pressure wave for a specific time window and then yield an 388 average blood pressure wave for the subject under consideration. Ideally, our algorithm could 389 be embedded into such a device and provide the additional approximation of arterial 390 compliance. In such a setting, a single carotid waveform would be sufficient. However, as 391 variations may occur across several beats of measurement, it is possible that the $\mathrm{C}_{\mathrm{T}}$ prediction 392 is influenced. Yet, the sensitivity analysis demonstrated that small alterations in the wave's 393 morphology due to noise do not affect significantly the predictions for an SNR equal to 40 $394 \mathrm{~dB}$. Validation of the methodology using multiple beats of carotid pressure remains to be 395 conducted in order to quantify the effect of such variations in vivo. 
The BP waveform has been shown to be crucial for assessing the vascular state in the human. It provides outstanding information on central hemodynamics, micro- and macrocirculation crosstalk, as well as arterial stiffness [2]-[4], [35], [36]. Moreover, signal processing techniques are rapidly advancing allowing for creating a gold mine of physiological information hidden in pressure blood waveform. In this study, we evaluated the performance of machine learning models on revealing the hidden information related to arterial compliance in commonly used pressure wave features. Furthermore, we tested the unrevealing capacity of an ANN which was fed with the raw pressure signal and received no guidance regarding the input features to use for the training/testing process. Interestingly, the algorithm performed very satisfactorily when the raw carotid waveform was prescribed to the ANN input layer. These findings indicate the beginning of a new era where the machine learning algorithms are capable of revealing more sophisticated piece of vascular information through learning by itself from the available clinical data.

Undoubtedly, noninvasive health monitoring technology is on the frontiers of modern healthcare and it is bound to expand inside and beyond the clinical environment. Concurrently, the rapid advance of wearable technologies is transforming the healthcare system on a global scale. Blood pressure sensing devices aim to be essentially miniaturized whereas their function will be highly assisted by pressure wave analysis techniques. In this context, reducing the required measurements to only a single waveform in conjunction with the greatly promising potential of signal processing techniques creates a unique opportunity for future use in the market. In addition, medical consultation is expected to become available remotely at all times by connecting to the data cloud where specialized clinicians will be interpreting the available parameters.

\section{Limitations}

A main limitation of this study is that the values for $\mathrm{C}_{\mathrm{T}}$, which were used as the ground truth, were derived using the PPM. Unarguably, this value does not correspond to the actual arterial compliance. Nonetheless, acquisition of the real arterial compliance would not be feasible in an intact organism. In addition, the PPM has been shown to provide reliable compliance estimations and, therefore, it constitutes a trustworthy alternative for validating our method [14], [15], [23]. Another limitation is that the study population included individuals free of cardiovascular disease or pathology. It is not guaranteed that the developed models will be capable of making predictions for patients with, for instance, aortic valve stenosis, arrhythmias or other pathologies. In future work, we aim to validate the proposed methodological framework using such populations. Finally, it is well established that healthy aging and cardiovascular diseases, such as hypertension and heart failure, are associated with increased arterial stiffness [37], [38]. Therefore, a method which is capable of differentiating between healthy and disease is highly desirable. At this initial stage, we demonstrated that accurate estimations of $\mathrm{C}_{\mathrm{T}}$ can be yielded using our ML-based approach. Given that $\mathrm{C}_{\mathrm{T}}$ has been found to be capable of differentiating between hypertensive, elderly and healthy individuals [10]-[12], as a next step, we envision to evaluate the robustness of the proposed method for classifying high-risk populations and finally verify its clinical significance in terms of risk stratification. 


\section{Conclusion}

441 This paper introduces a novel artificial intelligence method to estimate the $\mathrm{C}_{\mathrm{T}}$. The method relies on exploiting the information provided by the carotid blood pressure waveform as well as typical clinical variables (such as demographical data). Our results demonstrated that accurate estimates of $\mathrm{C}_{\mathrm{T}}$ can be obtained following our methodology. The importance of the method is based on the simplification of the technique offering easily applicable and convenient monitoring of $\mathrm{C}_{\mathrm{T}}$. Such an approach could offer promising applications which may be integrated in wearable technologies and smartphones. Finally, the study further supports the importance of arterial pulse waves in the assessment of cardiovascular health and suggests the potentiality of machine learning in advancing the detection of clinical biomarkers in medicine.

\section{Acknowledgments}

No acknowledgements.

455

456

\section{Disclosures}

No disclosures.

458

459

\section{References}

[1] W. Koenig, "Update on integrated biomarkers for assessment of long-term risk of cardiovascular complications in initially healthy subjects and patients with manifest atherosclerosis," Annals of Medicine, vol. 41, no. 5, pp. 332-343, Jan. 2009, doi: $10.1080 / 07853890902769675$.

[2] M. E. Safar and G. M. London, "Arterial and venous compliance in sustained essential hypertension.," Hypertension, vol. 10, no. 2, pp. 133-139, Aug. 1987, doi: 10.1161/01.HYP.10.2.133.

[3] D. Chemla, I. Antony, Y. Lecarpentier, and A. Nitenberg, "Contribution of systemic vascular resistance and total arterial compliance to effective arterial elastance in humans," American Journal of Physiology-Heart and Circulatory Physiology, vol. 285, no. 2, pp. H614-H620, Aug. 2003, doi: 10.1152/ajpheart.00823.2002.

[4] B. A. Haluska, L. Jeffriess, M. Downey, S. G. Carlier, and T. H. Marwick, "Influence of cardiovascular risk factors on total arterial compliance," Journal of the American Society of Echocardiography, vol. 21, no. 2, pp. 123-128, Feb. 2008, doi: 10.1016/j.echo.2007.05.043.

[5] G. Elzinga and N. Westerhof, "Pressure and Flow Generated by the Left Ventricle against Different Impedances," Circulation Research, vol. 32, no. 2, pp. 178-186, Feb. 1973, doi: 10.1161/01.RES.32.2.178.

[6] P. Segers et al., "Noninvasive (input) impedance, pulse wave velocity, and wave reflection in healthy middle-aged men and women," Hypertension, vol. 49, no. 6, pp. 1248-1255, Jun. 2007, doi: 10.1161/HYPERTENSIONAHA.106.085480.

[7] N. Stergiopulos, J. J. Meister, and N. Westerhof, "Determinants of stroke volume and systolic and diastolic aortic pressure," American Journal of Physiology-Heart and 
Circulatory Physiology, vol. 270, no. 6, pp. H2050-H2059, Jun. 1996, doi: 10.1152/ajpheart.1996.270.6.H2050.

[8] R. Heitmar, "Total arterial compliance: the future of cardiovascular risk assessment?," $J$ Hum Hypertens, vol. 24, no. 4, pp. 227-229, Apr. 2010, doi: 10.1038/jhh.2009.106.

[9] B. A. Haluska, L. Jeffries, S. Carlier, and T. H. Marwick, "Measurement of arterial distensibility and compliance to assess prognosis," Atherosclerosis, vol. 209, no. 2, pp. 474-480, Apr. 2010, doi: 10.1016/j.atherosclerosis.2009.10.018.

[10] L. Van Bortel and J. Spek, "Influence of aging on arterial compliance," J Hum Hypertens, vol. 12, no. 9, pp. 583-586, Sep. 1998, doi: 10.1038/sj.jhh.1000669.

[11] A. Beltran, "Arterial compliance abnormalities in isolated systolic hypertension," American Journal of Hypertension, vol. 14, no. 10, pp. 1007-1011, Oct. 2001, doi: 10.1016/S0895-7061(01)02160-4.

[12] L. Lind, "Arterial compliance and endothelium-dependent vasodilation are independently related to coronary risk in the elderly: the Prospective Investigation of the Vasculature in Uppsala Seniors (PIVUS) study," Clinical Physiology and Functional Imaging, vol. 28, no. 6, pp. 373-377, Nov. 2008, doi: 10.1111/j.1475097X.2008.00816.X.

[13] Z. Liu, K. P. Brin, and F. C. Yin, "Estimation of total arterial compliance: an improved method and evaluation of current methods," American Journal of Physiology-Heart and Circulatory Physiology, vol. 251, no. 3, pp. H588-H600, Sep. 1986, doi: 10.1152/ajpheart.1986.251.3.H588.

[14] N. Stergiopulos, P. Segers, and N. Westerhof, "Use of pulse pressure method for estimating total arterial compliance in vivo," Am. J. Physiol., vol. 276, no. 2 Pt 2, pp. H424-428, Feb. 1999.

[15] P. Segers et al., "Pulse pressure method and the area method for the estimation of total arterial compliance in dogs: sensitivity to wave reflection intensity," Ann Biomed Eng, vol. 27, no. 4, pp. 480-485, Aug. 1999.

[16] I. S. Mackenzie, I. B. Wilkinson, and J. R. Cockcroft, "Assessment of arterial stiffness in clinical practice," QJM, vol. 95, no. 2, pp. 67-74, Feb. 2002.

[17] N. Stergiopulos, J. J. Meister, and N. Westerhof, "Evaluation of methods for estimation of total arterial compliance," American Journal of Physiology-Heart and Circulatory Physiology, vol. 268, no. 4, pp. H1540-H1548, Apr. 1995, doi: 10.1152/ajpheart.1995.268.4.H1540.

[18] O. S. Randall, M. D. Esler, R. V. Calfee, G. F. Bulloch, A. S. Maisel, and B. Culp, "Arterial compliance in hypertension," Australian and New Zealand Journal of Medicine, vol. 6, pp. 49-59, Jun. 1976, doi: 10.1111/j.1445-5994.1976.tb03323.x.

[19] S. Sakuragi and W. P. Abhayaratna, "Arterial stiffness: methods of measurement, physiologic determinants and prediction of cardiovascular outcomes," Int. J. Cardiol., vol. 138, no. 2, pp. 112-118, Jan. 2010, doi: 10.1016/j.ijcard.2009.04.027.

[20] S. Laurent et al., "Expert consensus document on arterial stiffness: methodological issues and clinical applications," European Heart Journal, vol. 27, no. 21, pp. 25882605, Sep. 2006, doi: 10.1093/eurheartj/ehl254.

[21] K. Shameer, K. W. Johnson, B. S. Glicksberg, J. T. Dudley, and P. P. Sengupta, "Machine learning in cardiovascular medicine: are we there yet?," Heart, vol. 104, no. 14, pp. 1156-1164, Jul. 2018, doi: 10.1136/heartjnl-2017-311198.

[22] P. Tavallali, M. Razavi, and N. M. Pahlevan, "Artificial intelligence estimation of carotid-femoral pulse wave velocity using carotid waveform," Sci Rep, vol. 8, no. 1, p. 1014, Dec. 2018, doi: 10.1038/s41598-018-19457-0. 
532

533

534

535

536

537

538

539

540

541

542

543

544

545

546

547

548

549

550

551

552

553

554

555

556

557

558

559

560

561

562

563

564

565

566

567

568

569

570

571

572

573

574

575

576

577

578

579

580

[23] N. Stergiopulos, J. J. Meister, and N. Westerhof, "Simple and accurate way for estimating total and segmental arterial compliance: the pulse pressure method," Ann Biomed Eng, vol. 22, no. 4, pp. 392-397, Aug. 1994.

[24] E.-R. Rietzschel et al., "Rationale, design, methods and baseline characteristics of the Asklepios Study," Eur J Cardiovasc Prev Rehabil, vol. 14, no. 2, pp. 179-191, Apr. 2007, doi: 10.1097/HJR.0b013e328012c380.

[25] P. Segers et al., "Carotid Tonometry Versus Synthesized Aorta Pressure Waves for the Estimation of Central Systolic Blood Pressure and Augmentation Index," American Journal of Hypertension, vol. 18, no. 9, pp. 1168-1173, Sep. 2005, doi: 10.1016/j.amjhyper.2005.04.005.

[26] D. P. Kingma and J. Ba, "Adam: a method for stochastic optimization," presented at the 3rd International Conference for Learning Representations, San Diego, US, 2017. [Online]. Available: https://arxiv.org/pdf/1412.6980.pdf

[27] L. Breiman, "Random forests," Machine Learning, vol. 45, no. 1, pp. 5-32, 2001, doi: 10.1023/A:1010933404324.

[28] F. Pedregosa et al., "Scikit-learn: machine learning in Python," JMLR, vol. 12, pp. 2825-2830, 2011.

[29] W. McKinney, "Data structures for statistical computing in Python," in Proceedings of the 9th Python in Science Conference, 2010, pp. 51-56. doi: 10.25080/Majora92bf1922-00a.

[30] T. E. Oliphant, “A guide to NumPy,” vol. 1, Trelgol Publishing USA, 2006.

[31] J. M. Bland and D. G. Altman, "Statistical methods for assessing agreement between two methods of clinical measurement," Lancet, vol. 1, no. 8476, pp. 307-310, Feb. 1986.

[32] E. Litton* and M. Morgan, "The PiCCO Monitor: A Review," Anaesthesia and Intensive Care, vol. 40, no. 3, pp. 393-408, May 2012, doi: 10.1177/0310057X1204000304.

[33] J. D. Cameron, G. L. Jennings, and A. M. Dart, "Systemic arterial compliance is decreased in newly-diagnosed patients with coronary heart disease: implications for prediction of risk," J Cardiovasc Risk, vol. 3, no. 6, pp. 495-500, Dec. 1996.

[34] N. Stergiopulos and N. Westerhof, "Determinants of pulse pressure," Hypertension, vol. 32, no. 3, pp. 556-559, Sep. 1998, doi: 10.1161/01.HYP.32.3.556.

[35] B. A. Haluska, "Influence of arterial compliance on presence and extent of ischaemia during stress echocardiography," Heart, vol. 92, no. 1, pp. 40-43, Jan. 2006, doi: 10.1136/hrt.2004.052209.

[36] P. Bruinsma, T. Arts, J. Dankelman, and J. A. E. Spaan, "Model of the coronary circulation based on pressure dependence of coronary resistance and compliance," Basic Res Cardiol, vol. 83, no. 5, pp. 510-524, Sep. 1988, doi: 10.1007/BF01906680.

[37] G. F. Mitchell, "Arterial Stiffness and Hypertension: Chicken or Egg?," Hypertension, vol. 64, no. 2, pp. 210-214, Aug. 2014, doi: 10.1161/HYPERTENSIONAHA.114.03449.

[38] J. A. Chirinos, P. Segers, T. Hughes, and R. Townsend, "Large-Artery Stiffness in Health and Disease," Journal of the American College of Cardiology, vol. 74, no. 9, pp. 1237-1263, Sep. 2019, doi: 10.1016/j.jacc.2019.07.012. 
581

582

583

584

585

586

587

588

589

590

591

592

593

594

595

596

597

598

599

600

601

602

603

604

605

606

607

608

609

610

611

612

613

\section{Figure Legends}

Figure 1. Indication of features on the carotid pressure waveform and the time-derivative.

Figure 2. Comparison between predicted and reference data. Scatterplot and Bland-Altman plot between the predicted $\mathrm{C}_{\mathrm{T}}$ and the reference $\mathrm{C}_{\mathrm{T}}$ for LR1 (A), and LR2 (B). The solid line of the scatterplots represents equality. In Bland-Altman plots, limits of agreement (LoA) are defined by the two horizontal dashed lines.

Figure 3. Comparison between predicted and reference data. Scatterplot and Bland-Altman plot between the predicted $\mathrm{C}_{\mathrm{T}}$ and the reference $\mathrm{C}_{\mathrm{T}}$ for ANN1 (A), ANN2 (B), ANN3 (C), ANN4 (D). The solid line of the scatterplots represents equality. In Bland-Altman plots, limits of agreement (LoA) are defined by the two horizontal dashed lines.

Figure 4. Carotid blood pressure waves after adding artificial noise. The noisy data are presented in red solid lines and the original noise-free data in black dashed lines. 
Table 1. Asklepios inclusion and exclusion criteria.

\section{Inclusion criteria}

1 Male and female volunteers aged 35-55 years at study initiation, living in the communities of Erpe-Mere or Nieuwerkerken.

Exclusion criteria

1 Clinical presence of atherosclerosis/atherothrombosis

(a) Atherosclerosis: symptomatic or haemodynamically significant

( $>50 \%$ stenosis) presence of atherosclerosis in any major vascular bed.

(b) Atherothrombosis: acute coronary syndromes, cerebrovascular thrombosis.

(c) Previous or planned revascularization procedure (carotid, coronary, lower limb).

2 Major concomitant illness

(a) Cardiac: cardiomyopathy/heart failure, significant valvular disease, previous cardiac surgery, (complex) congenital heart disease, heart transplant.

(b) Organ failure: end-stage renal disease, hepatic insufficiency, previous organ transplant.

(c) Malignant tumours (recently diagnosed or currently treated, with $<3$ years tumour-free follow-up or tumours that are metastatic or initial treatment was not curative).

(d) Other conditions in which the screening physician expected a life expectancy $<5$ years.

3 Diabetes mellitus

(a) Diabetes mellitus type 1 .

(b) Diabetes mellitus type 2 if confirmed macrovasculopathy (see exclusion criterion 1) or significant renal impairment [see exclusion criterion 2(b)]. 4 Specific conditions precluding accurate haemodynamic assessment.

(a) Continually irregular cardiac cycle: atrial fibrillation.

(b) State of hyperdynamic activity: pregnancy (in the preceding 6 months).

5 Inability to provide informed consent. 
Table 2. Summary of all the ML models trained/tested based on their inputs.

\begin{tabular}{|c|c|c|}
\hline Model & Inputs & $\begin{array}{l}\text { Input vector } \\
\text { size }\end{array}$ \\
\hline LR1 & $\begin{array}{l}\text { SBP, DBP, MAP, PP, } P_{D N}, t_{D N}, A_{\text {upstroke }}, A_{\text {systolic }}, A_{\text {diastolic }} \\
\mathrm{dP} / \mathrm{dt}_{\max }, t_{d P / d t m a x}, H R \text {, age, gender, height, weight }\end{array}$ & 16 inputs \\
\hline LR2 & $\begin{array}{l}\mathrm{SBP}, \mathrm{DBP}, \mathrm{MAP}, \mathrm{PP}, \mathrm{P}_{\mathrm{DN}}, \mathrm{t}_{\mathrm{DN}}, \mathrm{A}_{\text {upstroke }}, \mathrm{A}_{\text {systolic }}, \mathrm{A}_{\text {diastolic }} \\
\mathrm{dP} \mathrm{dt}_{\mathrm{max}}, \mathrm{t}_{\mathrm{dP} / \mathrm{dtmax}}, \mathrm{HR} \text {, age, gender, height, weight, } \mathrm{CO}\end{array}$ & 17 inputs \\
\hline ANN1 & $\begin{array}{l}\text { SBP, DBP, MAP, PP, } \mathrm{P}_{\mathrm{DN}}, \mathrm{t}_{\mathrm{DN}}, \mathrm{A}_{\text {upstroke}}, \mathrm{A}_{\text {systolic }}, \mathrm{A}_{\text {diastolic }}, \\
\mathrm{dP} / \mathrm{dt}_{\max }, \mathrm{t}_{\mathrm{dP} / \mathrm{dtmax}}, \mathrm{HR} \text {, age, gender, height, weight }\end{array}$ & 16 inputs \\
\hline ANN2 & $\begin{array}{l}\mathrm{SBP}, \mathrm{DBP}, \mathrm{MAP}, \mathrm{PP}, \mathrm{P}_{\mathrm{DN}}, \mathrm{t}_{\mathrm{DN}}, \mathrm{A}_{\text {upstroke}}, \mathrm{A}_{\text {systolic }}, \mathrm{A}_{\text {diastolic }} \\
\mathrm{dP} / \mathrm{dt}_{\max }, \mathrm{t}_{\mathrm{dP} / \mathrm{dtmax}}, \mathrm{HR} \text {, age, gender, height, weight, } \mathrm{CO}\end{array}$ & 17 inputs \\
\hline ANN3 & $\begin{array}{l}\text { Entire raw carotid pressure waveform, HR, age, gender, } \\
\text { height, weight }\end{array}$ & 105 inputs \\
\hline ANN4 & Entire raw carotid pressure waveform, HR & 101 inputs \\
\hline ANN5 & $\begin{array}{l}\text { PP, SBP, } A_{\text {diastolic }}, A_{\text {systolic, weight (Most important }} \\
\text { features) }\end{array}$ & 5 inputs \\
\hline
\end{tabular}


Table 3. Optimal number of epochs for each ANN.

\begin{tabular}{|l|c|}
\hline & Epochs \\
\hline C $_{\text {T models }}$ & \\
\hline ANN1 & 118 \\
\hline ANN2 & 380 \\
\hline ANN3 & 187 \\
\hline ANN4 & 212 \\
\hline ANN5 & 101 \\
\hline
\end{tabular}


Table 4. Description of the cardiovascular characteristics and parameters of the study cohort.

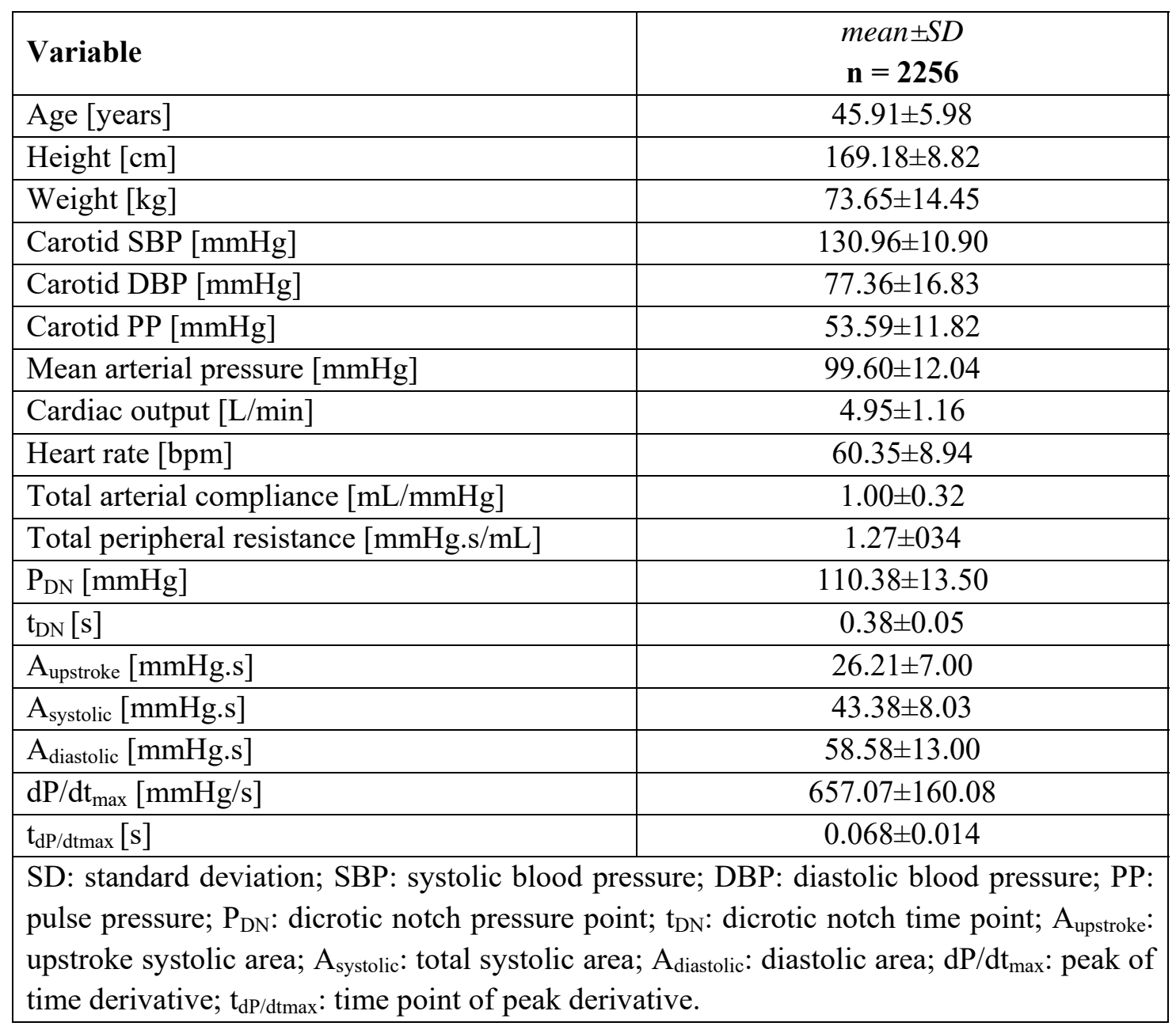


Table 5. Regression statistics between model-predicted and reference $\mathbf{C}_{\mathrm{T}}$ data.

\begin{tabular}{|l|c|c|c|c|c|c|c|}
\hline Model & Slope & $\begin{array}{c}\text { Intercept } \\
{[\mathbf{m L} / \mathbf{m m H g}]}\end{array}$ & $\mathbf{r}$ & $\mathbf{p}$-value & $\begin{array}{c}\text { nRMSE } \\
{[\mathbf{\%}]}\end{array}$ & $\begin{array}{c}\text { Bias } \mathbf{( L o A}) \\
{[\mathbf{m L} / \mathbf{m m H g}]}\end{array}$ & $\begin{array}{c}\text { Predicted } \mathbf{C}_{\mathbf{T}} \\
{[\mathbf{m L} / \mathbf{m m H g}]}\end{array}$ \\
\hline LR1 & 0.71 & 0.29 & 0.81 & $<0.0001$ & 10.63 & $-0.00(-0.35,0.35)$ & $0.98 \pm 0.28$ \\
\hline LR2 & 0.93 & 0.07 & 0.93 & $<0.0001$ & 6.13 & $0.01(-0.21,0.22)$ & $0.99 \pm 0.3$ \\
\hline ANN1 & 0.77 & 0.24 & 0.83 & $<0.0001$ & 9.58 & $0.01(-0.33,0.34)$ & $0.99 \pm 0.28$ \\
\hline ANN2 & 0.97 & 0.05 & 0.96 & $<0.0001$ & 4.8 & $0.01(-0.15,0.18)$ & $1 \pm 0.3$ \\
\hline ANN3 & 0.73 & 0.28 & 0.83 & $<0.0001$ & 9.67 & $0.02(-0.32,0.36)$ & $1 \pm 0.27$ \\
\hline ANN4 & 0.68 & 0.28 & 0.77 & $<0.0001$ & 11.26 & $-0.04(-0.43,0.35)$ & $0.94 \pm 0.27$ \\
\hline ANN5 & 0.74 & 0.26 & 0.82 & $<0.0001$ & 9.9 & $0.01(-0.34,0.36)$ & $0.99 \pm 0.27$ \\
\hline
\end{tabular}

r: Pearson's correlation coefficient; nRMSE: normalized root mean square error; LoA: limits of agreement. Two-sided p-value for a hypothesis test whose null hypothesis is that the slope is zero, using Wald Test with t-distribution of the test statistic. 
Table 6. Permutation feature importances for the ANN1.

\begin{tabular}{l|c|l|c} 
Feature & $\begin{array}{c}+\boldsymbol{\delta} \mathbf{R M S E} \\
{[\mathbf{m L} / \mathbf{m m H g}]}\end{array}$ & Feature & $\begin{array}{c}+\boldsymbol{\delta} \mathbf{R M S E} \\
{[\mathbf{m L} / \mathbf{m m H g}]}\end{array}$ \\
\hline $\mathrm{PP}$ & $0.31 \pm 0.04$ & $\mathrm{P}_{\mathrm{DN}}$ & $0.04 \pm 0.01$ \\
$\mathrm{SBP}$ & $0.28 \pm 0.02$ & $\mathrm{MAP}$ & $0.03 \pm 0.01$ \\
$\mathrm{~A}_{\text {diastolic }}$ & $0.24 \pm 0.02$ & $\mathrm{dP} / \mathrm{dt}_{\max }$ & $0.02 \pm 0.00$ \\
$\mathrm{~A}_{\text {systolic }}$ & $0.14 \pm 0.02$ & $\mathrm{HR}$ & $0.02 \pm 0.01$ \\
Weight & $0.11 \pm 0.01$ & $\mathrm{t}_{\mathrm{dP} / \mathrm{dmax}}$ & $0.02 \pm 0.01$ \\
DBP & $0.07 \pm 0.01$ & $\mathrm{Age}$ & $0.02 \pm 0.00$ \\
Height & $0.05 \pm 0.01$ & $\mathrm{t}_{\mathrm{DN}}$ & $0.01 \pm 0.00$ \\
Gender & $0.04 \pm 0.01$ & $\mathrm{~A}_{\text {upstroke }}$ & $0.01 \pm 0.01$ \\
\hline \hline
\end{tabular}

SBP: systolic blood pressure; DBP: diastolic blood pressure; $\mathrm{HR}$ : heart rate; $\mathrm{P}_{\mathrm{DN}}$ : dicrotic notch pressure point; $\mathrm{t}_{\mathrm{DN}}$ : dicrotic notch time point; $A_{\text {upstroke: upstroke systolic area; }}$ $\mathrm{A}_{\text {systolic: }}$ : total systolic area; $\mathrm{A}_{\text {diastolic }}$ : diastolic area; $\mathrm{dP} / \mathrm{dt}_{\max }$ : peak of time derivative; $t_{d P / d t m a x}$ : time point of peak derivative. 
Table 7. Correlation coefficients and nRMSE values as a function of the artificial noise level in the distorted carotid pressure waves for the ANN1, and ANN3 models.

\begin{tabular}{|l|c|c|c|}
\hline Model & & r & nRMSE (\%) \\
\hline & Noise level (\%) & & \\
\hline \multirow{3}{*}{ ANN1 } & \pm 5 & 0.79 & 10.73 \\
\cline { 2 - 4 } & \pm 7 & 0.78 & 11.11 \\
\cline { 2 - 4 } & \pm 10 & 0.71 & 13.51 \\
\hline \multirow{4}{*}{ ANN3 } & SNR (dB) & & \\
\cline { 2 - 4 } & 40 & 0.8 & 10.34 \\
\cline { 2 - 4 } & 35 & 0.79 & 10.76 \\
\hline
\end{tabular}



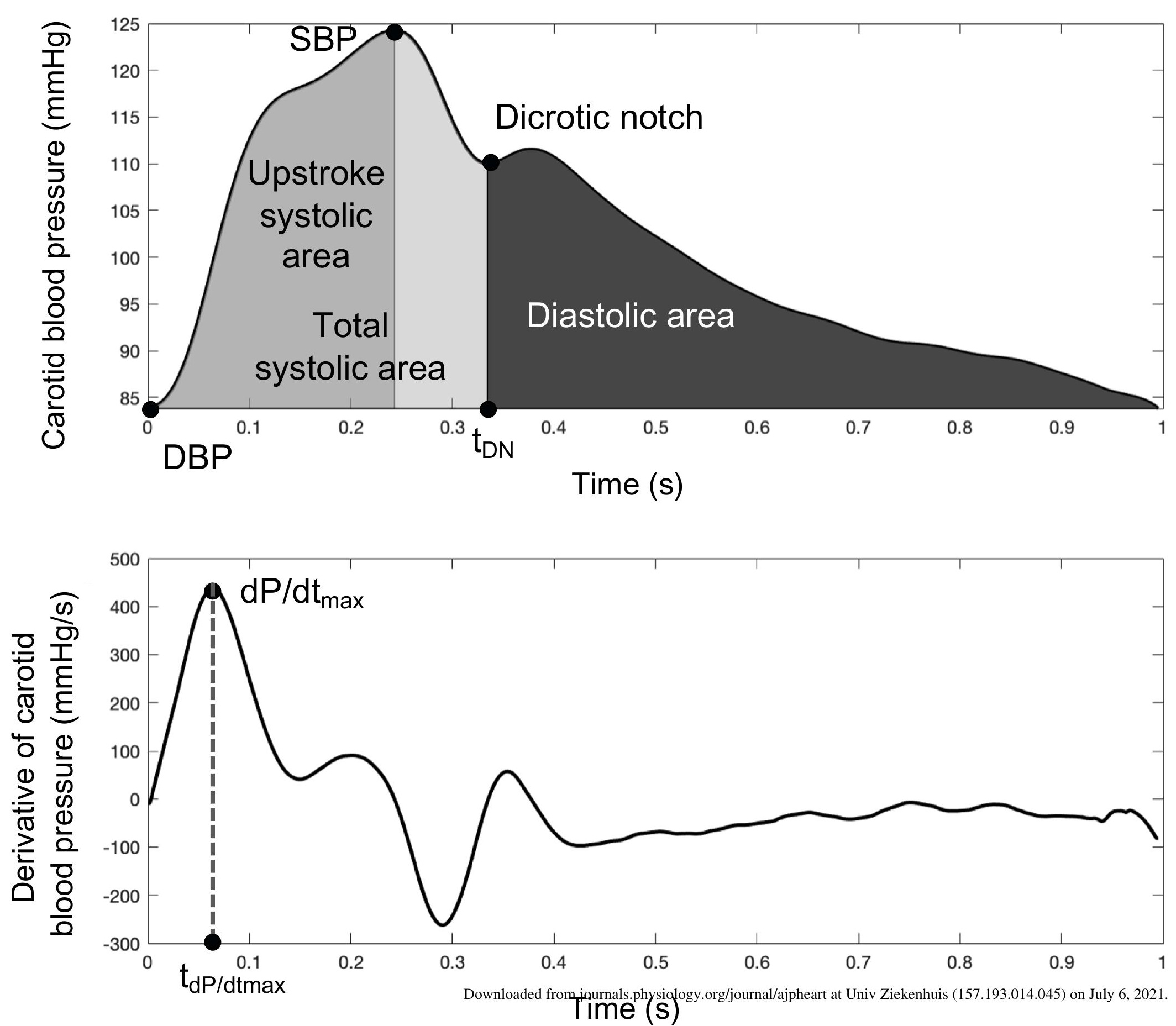
A
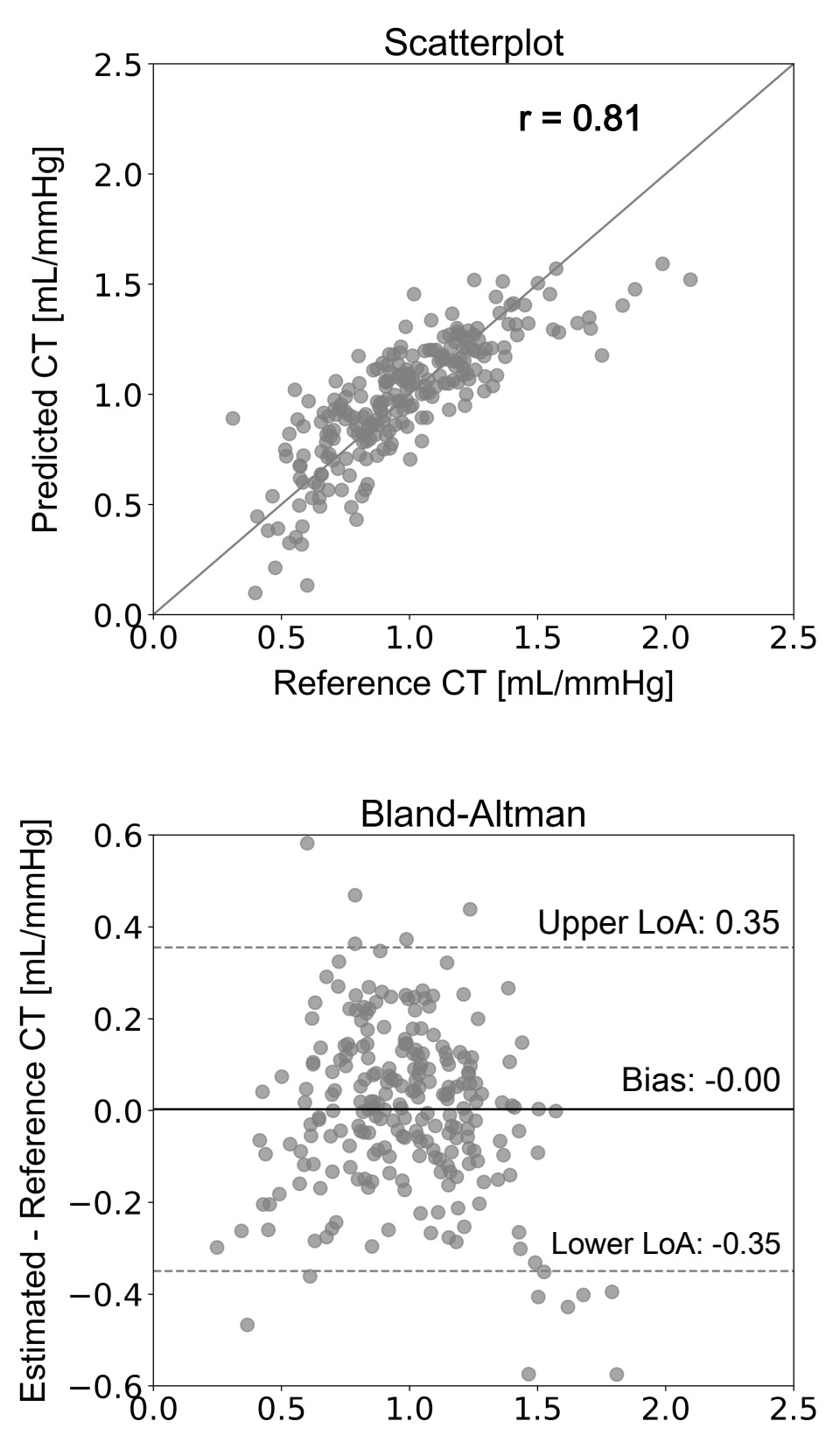

Mean of Reference and Estimated $\mathrm{CT}[\mathrm{mL} / \mathrm{mmH}$
B
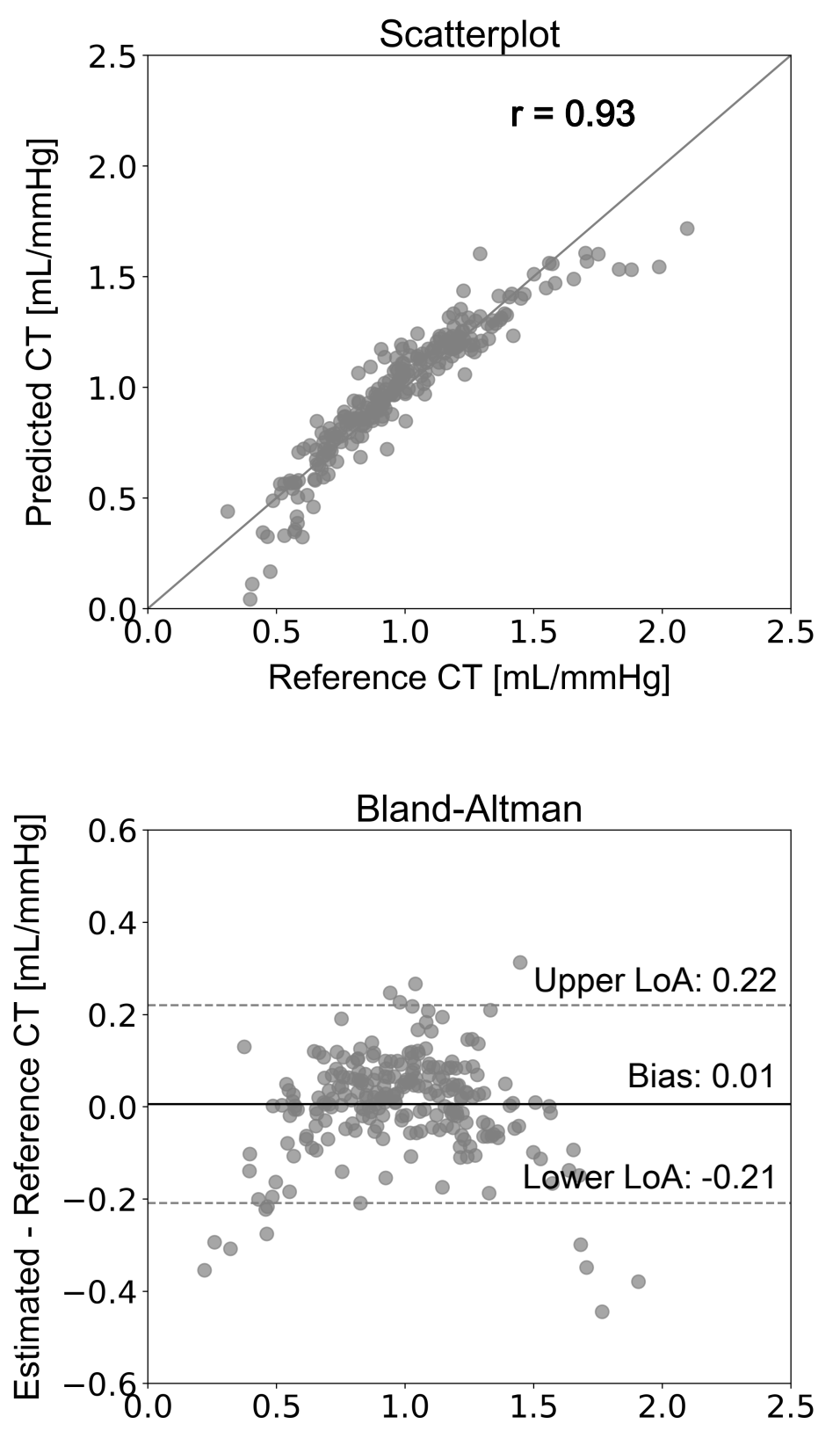
Downloaded from journals physiology org/journal/aipheart at Univ Ziekenhuis (157.193.014.045) on July 6, 2021 
A
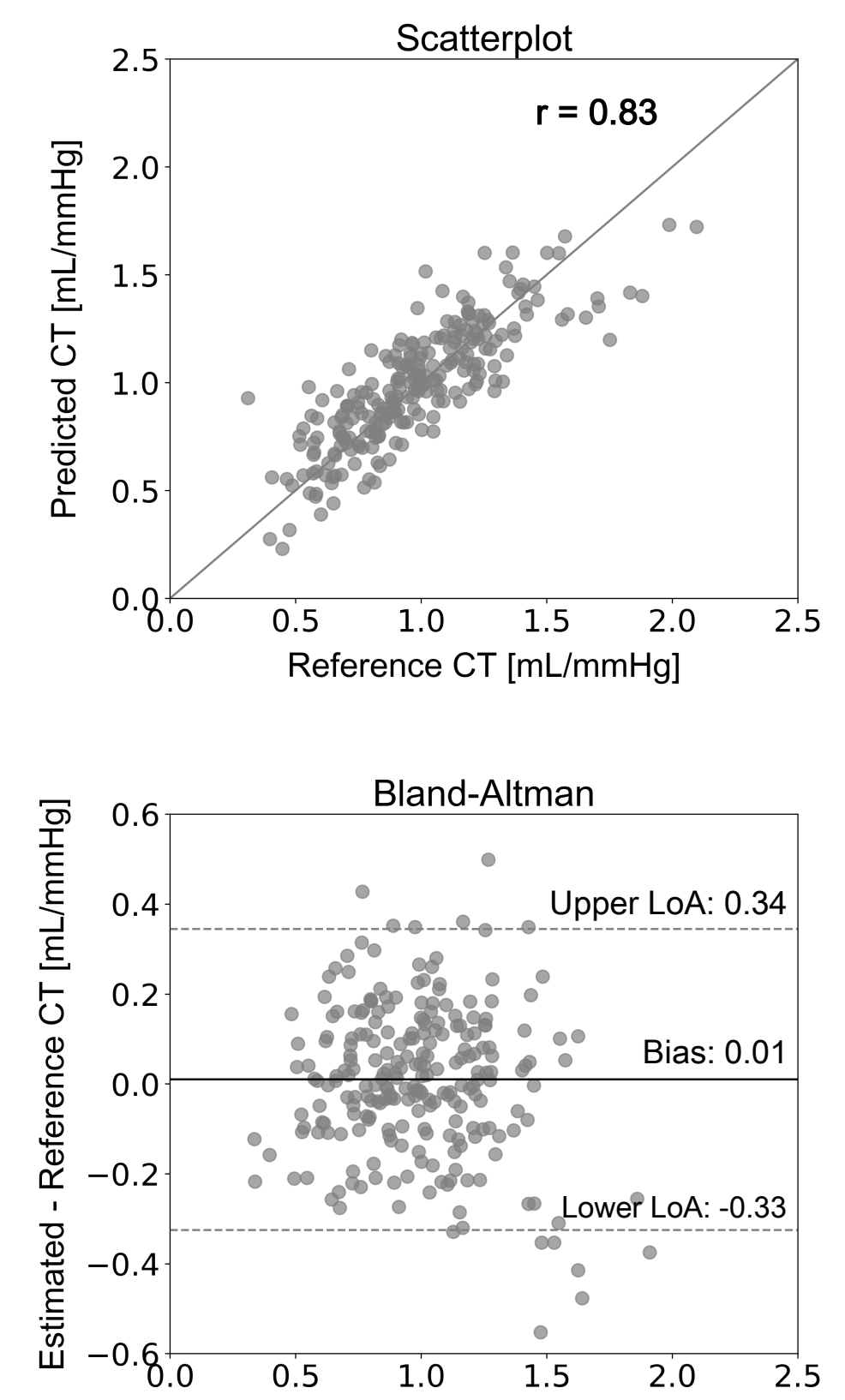

Mean of Reference and Estimated CT [mL/mmHg]
B
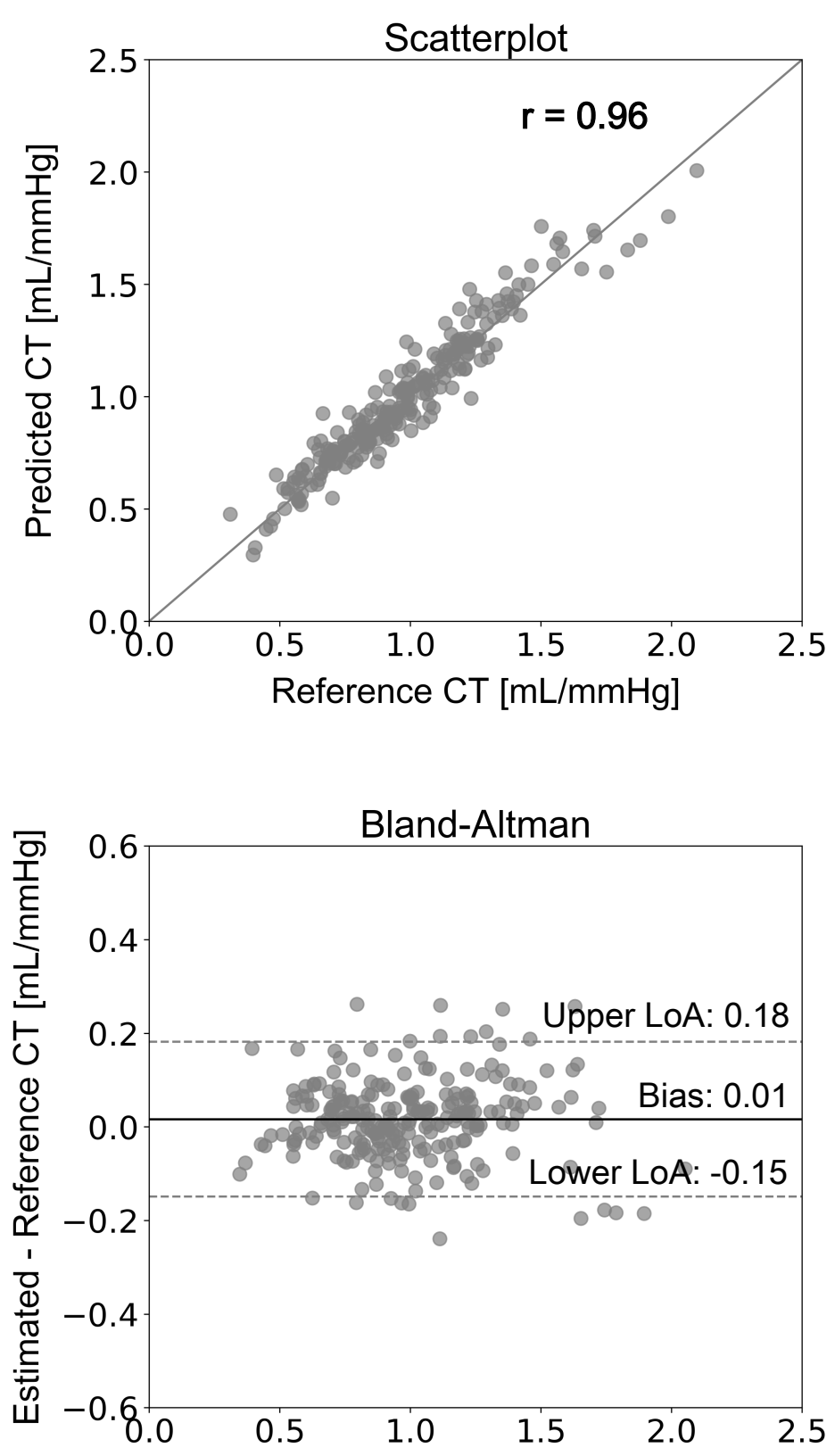
Downloaded from journals physiology org/journal/aipheart at Univ Ziekenhuis (157.193.014.045) on July 6, 202 


\section{C}
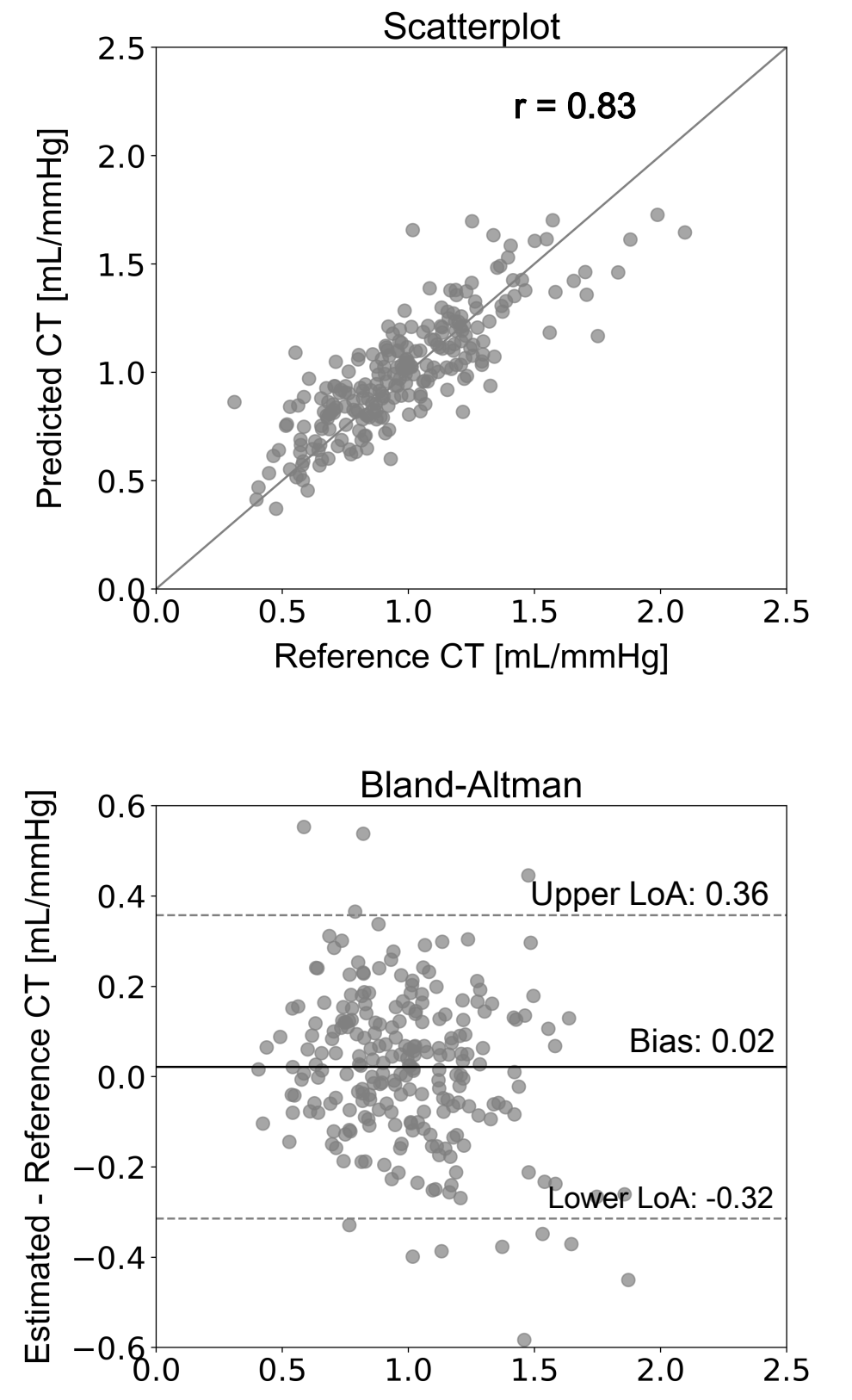

Mean of Reference and Estimated $\mathrm{CT}[\mathrm{mL} / \mathrm{mmHg}]$
D
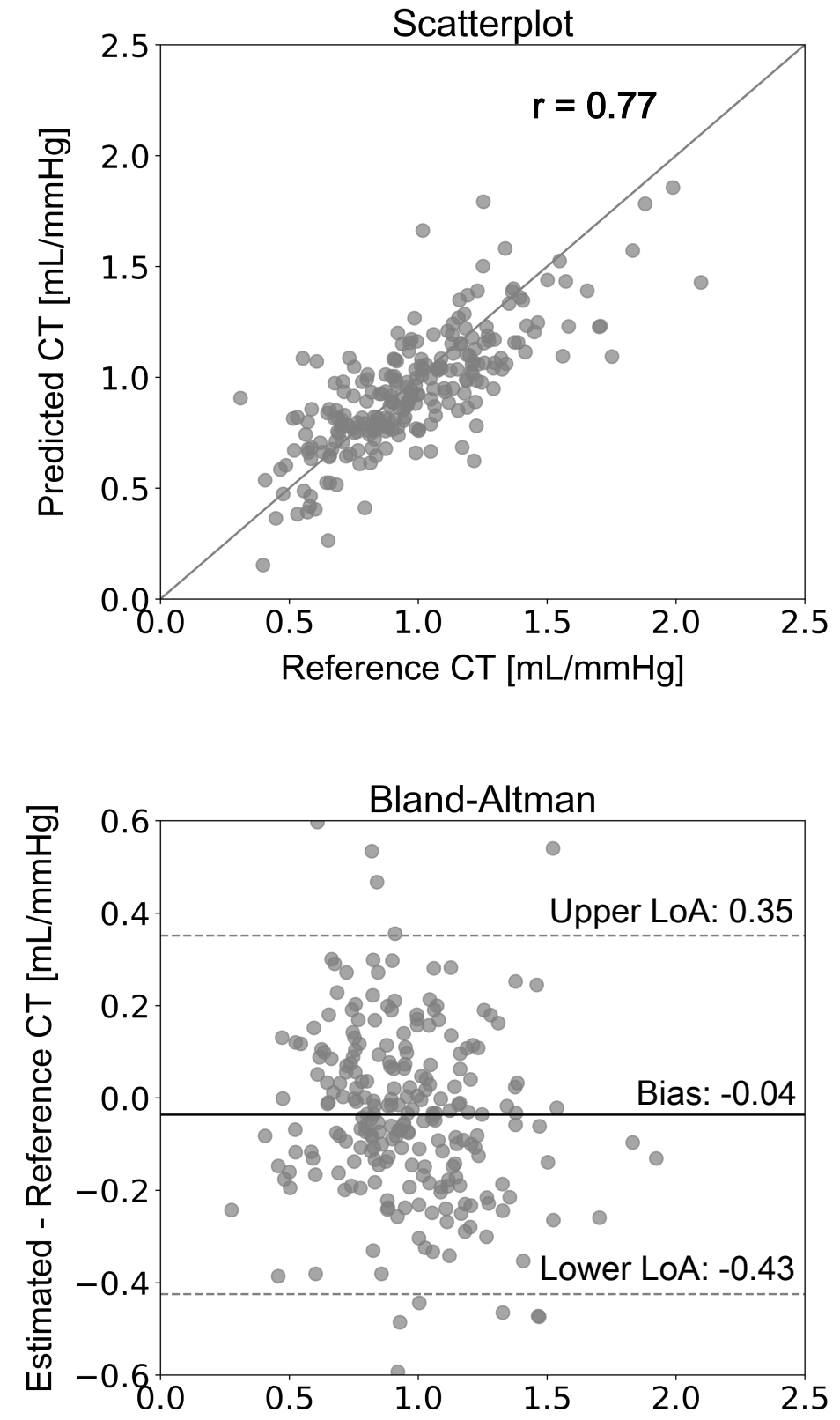
Downloaded from journals physiology org/journal/aipheart at Univ Ziekenhuis (157.193.014.045) on July 6, 2021. 


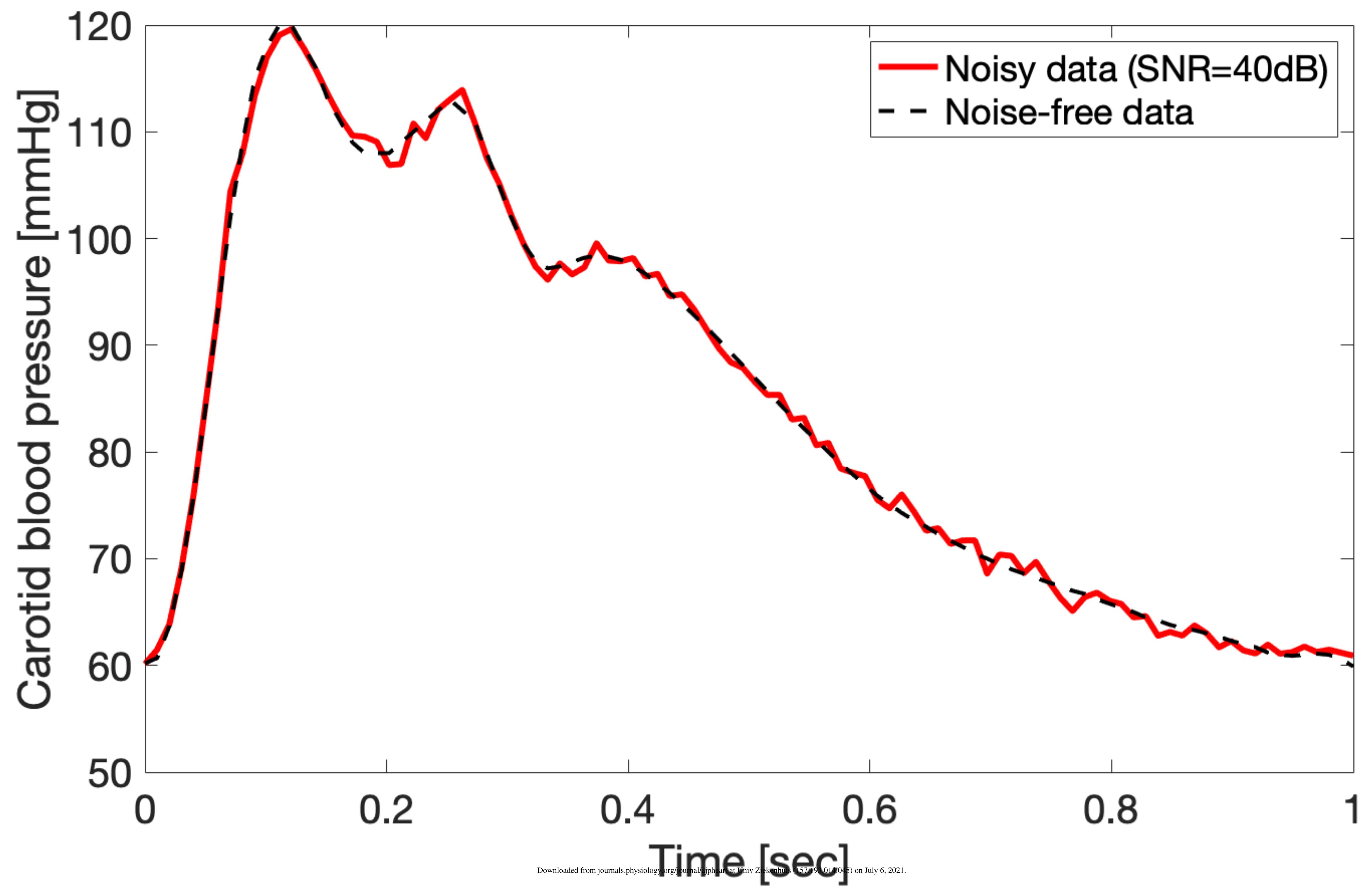




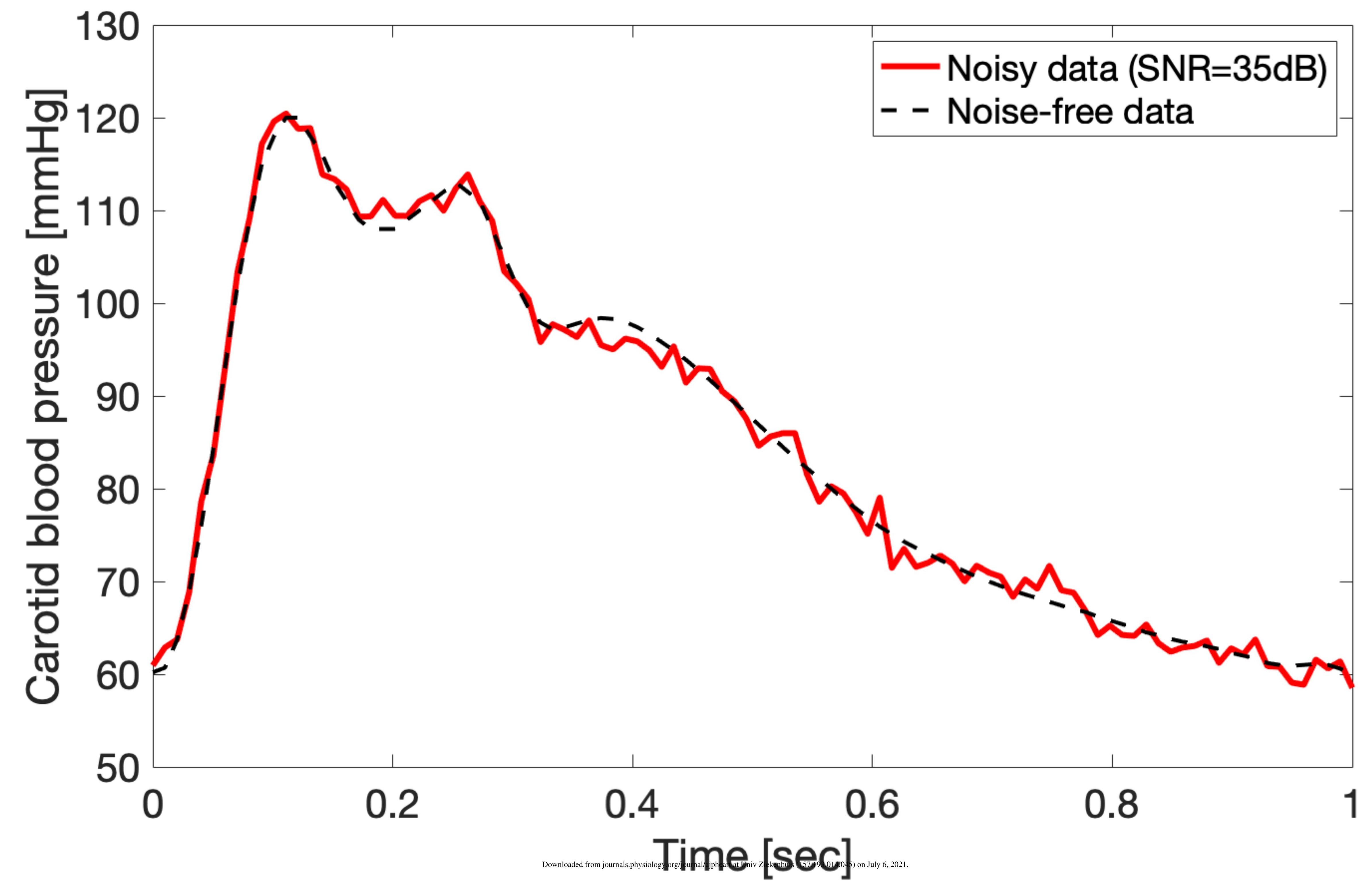




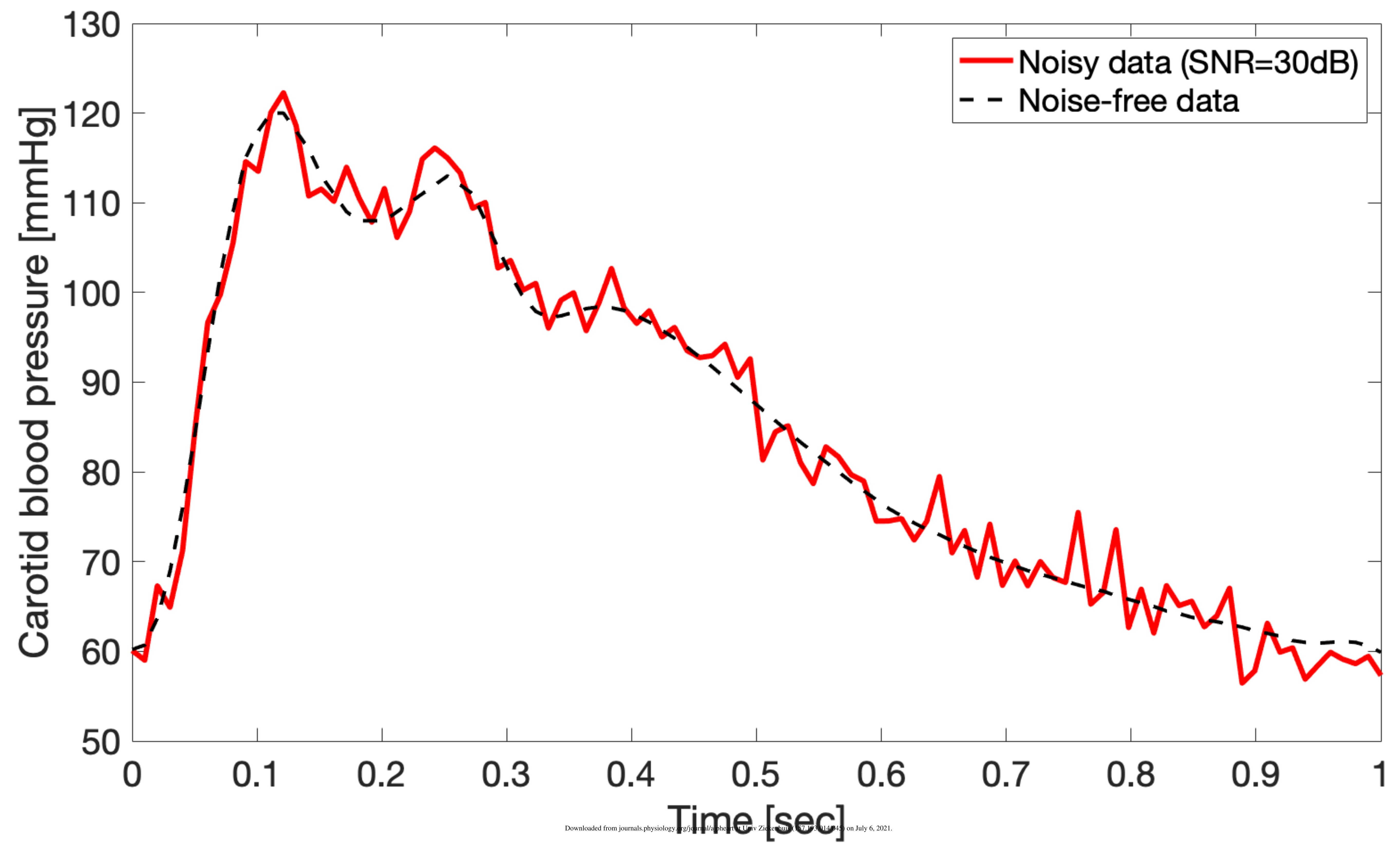

